\title{
Dialysate With High Dissolved Hydrogen Facilitates Dissociation of Indoxyl Sulfate From Albumin
}

\author{
Yoshihiro Tange ${ }^{1, *}$; Shingo Takesawa ${ }^{1}$; Shigenori Yoshitake ${ }^{1}$ \\ ${ }^{1}$ Department of Medical Engineering, School of Health Science, Kyushu University of Health and Welfare Yoshinomachi, Nobeoka, Japan \\ ${ }^{*}$ Corresponding author: Yoshihiro Tange, Department of Medical Engineering, School of Health Science, Kyushu University of Health and Welfare Yoshinomachi, Nobeoka, Miyazaki, \\ Japan. Tel/Fax:+81-982235592, E-mail: tan@phoenix.ac.jp
}

Received: January 10, 2015; Accepted: February 4, 2015

\begin{abstract}
Background: Protein-bound toxins such as indoxyl sulfate(IS)are not efficiently removed by conventional hemodialysis (HD).
Objectives: To improve the removal of IS, we performed an in vitro study to evaluate the effects of high dissolved hydrogen on the dissociation of IS from albumin using simulated HD.

Materials and Methods: Wasted dialysate from peritoneal dialysis was concentrated a hundred times using extracorporeal ultrafiltration method. Dialysate with high dissolved hydrogen was made by mixing concentrated dialysis solution and electrolyzed-reduced water. The amounts of free fractions of IS were determined by high performance liquid chromatography.

Results: IS was significantly dissociated from albumin using dialysate with high dissolved hydrogen compared with conventional dialysate $(\mathrm{P}<0.05)$.

Conclusions: Effective removal of IS is expected using a dialysate with high dissolved hydrogen.
\end{abstract}

Keywords:Dialysis Solutions; Hydrogen; Indoxyl Sulfate; Albumins

\section{Background}

High flux membranes have been applied for hemodialysis (HD). High flux dialysis membrane is defined as a $\beta 2$-microglobulin clearance of over $20 \mathrm{~mL} / \mathrm{min}(1,2)$. It is able to remove low molecular weight proteins between 20000 and 30000 Da. However, smaller molecules of uremic toxin connect albumin (molecular weight $=66,000$ ) are difficult to separate protein-bound toxins from albumin, because albumin molecular weight is high. Indoxyl sulfate (IS) is one of protein-bound toxins (3) known to release reactive oxygen species (ROS) to the blood stream and causes some dialysis-related complications. Therefore, it might be better if IS is dissociated from albumin and excluded during HD therapy. Yamamoto, et al. demonstrated in vitro that dilution and $\mathrm{pH}$ change of dialysate facilitate IS dissociate from albumin (4). The predilution mode can dilute the blood before hemodia filter in on-line HDF therapy. This method also facilitates the dissociation of IS from albumin by dilution and removal of dissociated IS (molecular weight $=213$ ) by diffusion.

Recent basic and clinical researches have revealed that hydrogen is an important physiological regulatory factor with antioxidant, anti-inflammatory and anti-apoptotic protective effects on cells and organs (5). A water electrolysis technique has been developed (6) and enabled to apply high dissolved hydrogen solution to clinical HD system. Use of dialysate with high dissolved hydrogen may prove to be a novel approach for amelioration of dialysis-related complications such as intradialytic hypotension (7).

\section{Objectives}

We made dialysate with high dissolved hydrogen. We examined in vitro whether IS dissociated from albumin in simulated HD system using this dialysate.

\section{Materials and Methods}

\subsection{Characteristic of Dialysate With High Dissolved Hydrogen}

We obtained high dissolved hydrogen solution by ion water conditioner (NIHON TRIM, Japan). To determine the characteristics of this solution, we used oxidationreduction potential (ORP) meter (YK- 23RP, Mother Tool, Japan), and hydrogen monitor (ENH-1000, TRUSTLEX, Japan). Sampling time was set at 0 minute as baseline, 30 minutes, 60 minutes, 120 minutes, 180 minutes, 240 minutes, 360 minutes, 12 hours and 24 hours after making this solution. Measurements were repeated five times.

\subsection{How to Make Conventional Dialysate and Di- alysate With High Dissolved Hydrogen}

We made conventional dialysate and the dialysate with

Copyright (C) 2015, Nephrology and Urology Research Center. This is an open-access article distributed under the terms of the Creative Commons Attribution-NonCommercial 4.0 International License (http://creativecommons.org/licenses/by-nc/4.0/) which permits copy and redistribute the material just in noncommercial usages, provided the original work is properly cited. 
high dissolved hydrogen using reverse osmosis (RO) water and high dissolved hydrogen solution, respectively and diluted 35 times with concentrated dialysate (KINDARY AF- 2, FUSO, Japan). pH, partial pressure of carbon dioxide (PCO2), oxygen (PO2) and $\mathrm{HCO} 3$ of conventional dialysate and high dissolved hydrogen dialysate were determined using i-STAT (300F, FUSO, Japan).

\subsection{Examination of Indoxyl Sulfate Separated From Albumin Using Dialysate With High Dis- solved Hydrogen}

We obtained $100 \mathrm{~L}$ wasted dialysate after continuous ambulatory peritoneal dialysis (CAPD) in a patient. We concentrated CAPD solution 100 times by extracorporeal ultrafiltration method using FB-190E (CTA, low-flux, NIPRO, Japan). One hundred $\mathrm{mL}$ of this solution was mixed with $100 \mathrm{~mL}$ of conventional dialysate or dialysate with high dissolved hydrogen dialysate. Experiment was set at blood flow rate of $100 \mathrm{~mL} / \mathrm{min}$, filtration flow of $5 \mathrm{~mL} / \mathrm{min}$ and dialyzer FB-70E (CTA, low-flux, NIPRO, Japan) as a filter to separate free IS and albumin-bound IS. We obtained $100 \mathrm{~mL}$ filtrated dialysate. The concentration of IS was determined by high performance liquid chromatography. Free fractions (FR) were calculated as below formula (4).

$$
\mathrm{FR}=\frac{C_{F}}{C_{B}} \times 100
$$

CB: initial concentration of blood side

CF: concentration of filtration side

\subsection{Statistical Analysis}

Statistical analyses were conducted using Stat View (Version 5, SAS, USA). Continuous variables were described as mean \pm standard deviation (SD) and compared with repeated measure ANOVA of Tukey-Kramer or Student's t-test as appropriate. P value less than 0.05 or 0.01 was regarded as significant.

\section{Results}

Dissolved hydrogen concentration and ORP in the electrolyzed solution in the high dissolved hydrogen solution. Changes in dissolved hydrogen and ORP after making the solution are shown in Figure 1. Dissolved hydrogen decreased half values compared at baseline in 120 minutes. Time course of ORP gradually increased. These results suggested that the half-life of hydrogen water was 120 minutes; the experiment was performed using electrolyzed solution in the high dissolved hydrogen within 30 minutes after making.

Solute characteristics of both dialysate profiles are shown in Table 1. Dissolved hydrogen showed significantly higher than conventional dialysate $(\mathrm{P}<0.01)$. Comparison between conventional dialysate and high dissolved hydrogen in the initial and filtrated concentrations of albumin IS and FR is shown in Table 2. FR of conventional dialysate showed $35.4 \pm 4.0 \%$. FR of high dissolved hydrogen showed $41.7 \pm 2.6 \%$. FR of high dissolved hydrogen was significantly higher than conventional dialysate $(\mathrm{P}<0.05)$.

Figure 1. Changes in Dissolved Hydrogen and ORP After Making the Solution

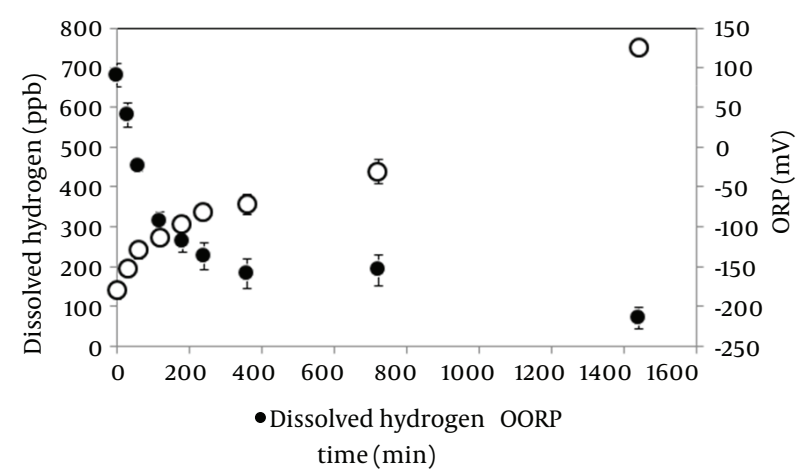

All data significantly changed $(\mathrm{P}<0.01)$ compared with baseline. $(\mathrm{n}=5$ Mean \pm SD).

Table 1. Profile of Conventional Dialysate and High Dissolved Hydrogen Dialysate $(n=5)^{a}$

\begin{tabular}{lccc}
\hline Variables & Conventional Dialysate & High H2 Dialysate & P Value \\
\hline $\mathbf{p H}$ & $7.59 \pm 0.18$ & $7.60 \pm 0.06$ & $\mathrm{~ns}$ \\
$\mathbf{P C O}_{\mathbf{2}}, \mathbf{m m H g}$ & $27.6 \pm 8.92$ & $27.3 \pm 2.87$ & $\mathrm{~ns}$ \\
$\mathbf{P O}_{\mathbf{2}}, \mathbf{m m H g}$ & $144.6 \pm 14.57$ & $134 \pm 20.22$ & $\mathrm{~ns}$ \\
$\mathbf{H C O}_{\mathbf{3}}, \mathbf{m m o l} / \mathrm{L}$ & $25.4 \pm 1.6$ & $26.7 \pm 1.0$ & $\mathrm{~ns}$ \\
\hline Dissolved & $\mathbf{2}, \mathbf{p p b}$ & $699.8 \pm 179.9$ & $<0.01$ \\
\hline
\end{tabular}

\footnotetext{
a All of the values are presented as Mean \pm SD.
} 
Table 2. Comparison of Initial and Filtrated Concentrations of Albumin, IS, and FR in Conventional Dialysate With High Dissolved Hydrogen Dialysate $(n=5)^{a, b}$

\begin{tabular}{lccc}
\hline Variables & Conventional Dialysate & High H2 Dialysate & PValue \\
\hline Initial concentration of albumin, $\boldsymbol{\mu} \mathbf{g} / \mathbf{m L}$ & $7569 \pm 175$ & $7527 \pm 310$ & $\mathrm{~ns}$ \\
Concentration of filtrated albumin, $\boldsymbol{\mu} \mathbf{g} / \mathbf{m L}$ & $39.1 \pm 3.6$ & $38.8 \pm 5.4$ & $\mathrm{~ns}$ \\
\hline Initial concentration of IS, $\boldsymbol{\mu} / \mathbf{m L}$ & $8.3 \pm 0.6$ & $8.4 \pm 0.5$ & $\mathrm{~ns}$ \\
Concentration of filtrated $\mathbf{I S}, \boldsymbol{\mu} \mathbf{g} / \mathbf{m L}$ & $2.9 \pm 0.4$ & $3.5 \pm 0.1$ & $<0.05$ \\
FR of IS, \% & $35.4 \pm 0.4$ & $41.7 \pm 2.7$ & $<0.05$ \\
\hline
\end{tabular}

a All values are presented as Mean \pm SD.

b Abbreviations: FR, Free Fractions; IS, Indoxyl Sulfate.

\section{Discussion}

Protein-bound uremic toxins, especially IS, induce vascular inflammation, endothelial dysfunction and vascular calcification, which may explain the relatively poor prognosis of chronic kidney disease and patients under dialysis (8). Plasma IS was associated with first heart failure event in patients on HD (9). HD using a high-flux membrane cannot efficiently remove the protein-bound uremic toxins because of their high albumin-binding property. Especially, IS showed high protein-binding ratios (more than 95\%) and low reduction rates by HD (< $35 \%$ ). Removal of IS can be improved to some extent by increasing the diffusion of free forms with super-flux membrane HD and/or HDF (10). IS is largely albumin bound and inhibits drug protein biding (11-14). Furthermore, this compound accelerates the progression of glomerulosclerosis in the rat $(15,16)$. If IS dissociates from albumin, it can easily remove by diffusion. Thus, IS removal is expected as new dialysis techniques. We focused on dialysate with high dissolved hydrogen and examined characteristics in this solution.

The level of dissolved hydrogen values was halved by two hours after obtaining electrolyzed water. These results suggested that the solution should be used immediately after preparation. Anti-oxidation has been confirmed using electrolyzed water by animal experiments (17-20). Furthermore, hydrogen gas has been reported to exhibit antioxidant properties. Electrolytic water containing hydrogen attracted more attention recently (21). Consumption of water with dissolved hydrogen produced by electrolysis by ad libitum drinking has the potential to ameliorate ischemia-induced cardio-renal injury in chronic kidney disease model rats (22). Using hydrogen-enriched solutions could ameliorate oxidative stress and albumin redox during HD (23).

Therefore, we focused on IS and developed a dialysate containing high dissolved hydrogen. We examined in vitro whether it is possible to separate IS from albumin. IS can be easily separated by dilution. This study demonstrated that hydrogen water promoted IS to dissociate from albumin during HD therapy. More beneficial effects would be expected in the combination of predilution mode on-line HDF with hydrogen water. More clinical studies are necessary on this issue. Dialysate with high dissolved hydrogen can significantly dissociate IS from albumin compared to conventional dialysate. Using this solution, high efficient IS removal was proved in vitro.

\section{Acknowledgements}

We acknowledge all staff of Ogawa Clinic for their cooperation in this study.

\section{Authors' Contributions}

Study concept and design: Yoshihiro Tange and Shigenori Yoshitake; Acquisition of data: Yoshihiro Tange; Analysis and interpretation of data: Yoshihiro Tange; Drafting of the manuscript: Yoshihiro Tange and Shigenori Yoshitake; Critical revision of the manuscript for important intellectual content: Yoshihiro Tange, Shingo Takesawa and Shigenori Yoshitake; Statistical analysis: Yoshihiro Tange; Administrative, technical and material supports: Shingo Takesawa and Shigenori Yoshitake; Study supervision: Shingo Takesawa and Shigenori Yoshitake.

\section{References}

1. Cheung AK, Leypoldt JK. The hemodialysis membranes: a historical perspective, current state and future prospect. Semin Nephrol. 1997;17(3):196-213

2. Leypoldt JK, Cheung AK, Agodoa LY, Daugirdas JT, Greene T, Keshaviah PR. Hemodialyzer mass transfer-area coefficients for urea increase at high dialysate flow rates. The Hemodialysis (HEMO) Study. Kidney Int. 1997;51(6):2013-7.

3. Vanholder R, Glorieux G, De Smet R, Lameire N, European Uremic Toxin Work G. New insights in uremic toxins. Kidney Int Suppl. 2003(84):S6-10.

4. Yamamoto K, Eguchi K, Kaneko I, Akiba T, Mineshima M. In vitro study of removal of protein-bound toxins. Blood Purif. 2013;35 Suppl 1:51-4.

5. Huang CS, Kawamura T, Toyoda Y, Nakao A. Recent advances in hydrogen research as a therapeutic medical gas. Free Radic Res. 2010;44(9):971-82.

6. Nakayama M, Kabayama S, Terawaki H, Nakayama K, Kato K Sato $\mathrm{T}$, et al. Less-oxidative hemodialysis solution rendered by cathode-side application of electrolyzed water. Hemodial Int. 2007;11(3):322-7.

7. Nakayama M, Kabayama S, Nakano H, Zhu WJ, Terawaki H, Nakayama K, et al. Biological effects of electrolyzed water in hemodialysis. Nephron Clin Pract. 2009;112(1):c9-15.

8. Ito S, Yoshida M. Protein-bound uremic toxins: new culprits of 
cardiovascular events in chronic kidney disease patients. Toxins (Basel). 2014;6(2):665-78.

9. Cao XS, Chen J, Zou JZ, Zhong YH, Teng J, Ji J, et al. Association of Indoxyl Sulfate with Heart Failure among Patients on Hemodialysis. Clin J Am Soc Nephrol. 2015;10(1):111-9.

10. Niwa T. Removal of protein-bound uraemic toxins by haemodialysis. Blood Purif. 2013;35 Suppl 2:20-5.

11. Vanholder R, De Smet R, Hsu C, Vogeleere P, Ringoir S. Uremic toxicity: the middle molecule hypothesis revisited. Semin Nephrol. 1994;14(3):205-18.

12. Niwa T. Organic acids and the uremic syndrome: protein metabolite hypothesis in the progression of chronic renal failure. Semin Nephrol.1996;16(3):167-82.

13. Niwa T, Yazawa T, Ise M, Sugano M, Kodama T, Uehara Y, et al. Inhibitory effect of oral sorbent on accumulation of albuminbound indoxyl sulfate in serum of experimental uremic rats. Nephron. 1991;57(1):84-8.

14. Bowmer CJ, Lindup WE. Decreased drug binding in uraemia: effect of indoxyl sulphate and other endogenous substances on the binding of drugs and dyes to human albumin. Biochem Pharmacol.1982;31(3):319-23.

15. Niwa T, Ise M, Miyazaki T. Progression of glomerular sclerosis in experimental uremic rats by administration of indole, a precursor of indoxyl sulfate. Am J Nephrol. 1994;14(3):207-12.

16. Niwa T, Ise M. Indoxyl sulfate, a circulating uremic toxin, stimulates the progression of glomerular sclerosis. J Lab Clin Med. 1994;124(1):96-104.
17. Nakayama M, Nakano H, Hamada H, Itami N, Nakazawa R, Ito S. A novel bioactive haemodialysis system using dissolved dihydrogen $(\mathrm{H} 2)$ produced by water electrolysis: a clinical trial. Nephrol Dial Transplant. 2010;25(9):3026-33.

18. Shirahata S, Kabayama S, Nakano M, Miura T, Kusumoto K, Gotoh $\mathrm{M}$, et al. Electrolyzed-reduced water scavenges active oxygen species and protects DNA from oxidative damage. Biochem Biophys Res Commun. 1997;234(1):269-74.

19. Li Y, Nishimura T, Teruya K, Maki T, Komatsu T, Hamasaki T, et al. Protective mechanism of reduced water against alloxan-induced pancreatic beta-cell damage: Scavenging effect against reactive oxygen species. Cytotechnology. 2002;40(1-3):139-49.

20. Li Y, Hamasaki T, Nakamichi N, Kashiwagi T, Komatsu T, Ye J, et al. Suppressive effects of electrolyzed reduced water on alloxaninduced apoptosis and type 1 diabetes mellitus. Cytotechnology. 2011;63(2):119-31.

21. Ohsawa I, Ishikawa M, Takahashi K, Watanabe M, Nishimaki K, Yamagata $\mathrm{K}$, et al. Hydrogen acts as a therapeutic antioxidant by selectively reducing cytotoxic oxygen radicals. Nat Med. 2007;13(6):688-94.

22. Zhu WJ, Nakayama M, Mori T, Nakayama K, Katoh J, Murata Y, et al. Intake of water with high levels of dissolved hydrogen $(\mathrm{H} 2)$ suppresses ischemia-induced cardio-renal injury in Dahl saltsensitive rats. Nephrol Dial Transplant. 2011;26(7):2112-8.

23. Terawaki H,Zhu WJ, Matsuyama Y, Terada T, Takahashi Y, Sakurai K, et al. Effect of a hydrogen (H2)-enriched solution on the albumin redox of hemodialysis patients. Hemodial Int. 2014;18(2):459-66. 